
\title{
$q u$
}

新

SLAC-PUB- -4187

DE87 010541

SLAC - PUB - 4187

SLAC/AP - 55

January 1987

A/AP

\section{CHANNELED PARTICLE ACCELERATION BY PLASMA WAVES IN METALS}

\author{
PISIN CHEN* \\ Stanford Linear Accelerstor Center, Stanford, CA 94305 \\ and \\ ROBERT J. NOBLE \\ Fermi National Accelerator Laboratory, Batavia, IL $60510^{\dagger}$
}

\begin{abstract}
We present a solid state accelerator concept utilizing particle acceleration along crystal channelo by longitudinal electron plasma weves in a metal. Acceleration gradients of order $100 \mathrm{GV} / \mathrm{cm}$ are theoretically possible. Particle dechanneling due to electron multiple acattering can be ellminated with a sufficlently high acceleration gradient. Plasma wave dissipation and generation in metals are also discussed.
\end{abstract}

Contributed to Relativistic Channeling

|Acquafredda di Maratea, ttaly (March 31-April 4, 1986),

R. A. Cartigan, Jr. and J. Ellison, edg. NATO ASI Series (Plenum)|.

\footnotetext{
-Work supported in part by the Department of Energy, contract DE-AC03765 Fo0515.

TOperated by Universities Research Association, Inc., under contract with the v. 5. Department of Energy.
} 


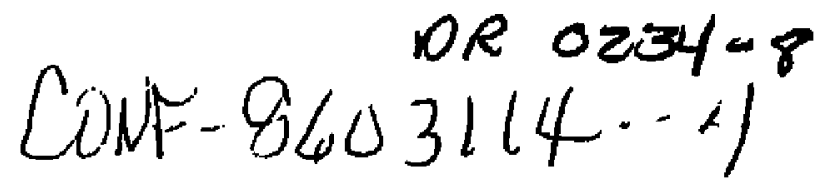

Presently existing high encrgy particle accelerators are limited to accelera. tion gradients of order $10 \mathrm{MV} /$ meter. This implies that to achieve ultrahigh energies exceeding several TeV would require great distances. In tecent years there has been an increased intercst in the high-gradicnt linear acceleration of changed particles,", One concept which promises very high gradients is the plasma accelerator. ${ }^{3}$ In thie scheme tongitudinal plasma oscillations with phase velocities near the speed of light provide large electric ficlds which are intended to accelerate particles to high energy over a short distance. Gradients $G$ of order $\sqrt{n} \mathrm{~V} / \mathrm{cm}$ are theoretically possible, where $n$ is the electron number density in units of $\mathrm{cm}^{-3}$. Typical laboratory gas plasma densities are in the range $10^{14}-10^{18}$ $\mathrm{cm}^{-3}$ corresponding to maximum gradients of $10 \mathrm{MV} / \mathrm{cm}-1 \mathrm{GV} / \mathrm{cm}$.

However, a high gradient is not the only requirement for linear colliders. Stability and emittance requirements for the secelerating system are very stringant. Since the beams from two independent accelerators must collide at an interaction point, excessive transverse motion and emitlance growth of the beams induced during acceleration must be avoided. One concern is that plasma accelerators may be prone to such beam instabilities due to plasma nonuniformities and multiple ion scattering.

To extend the plasma wave acceleration idea to very high gradients and reduce emittance growth, we explore in this paper a solid state accelerator concept in which particles arc accelerated along atomic erystal channels by plasma waves in a metal. Conduction electrons in a metal form a very uniform high density plasma exhibiting longitudinal plasma oscillations.' Typical conduction electron densities are of order $10^{73} \mathrm{~cm}^{-3}$ corrosponding to a maximum gradient of order $100 \mathrm{GV} / \mathrm{cm}$. Although this gradient equals $10^{3} \mathrm{~V} / \mathrm{A}$, the metal can support such high fields becauge the ionization cnergy of the atomic core electrons is at least several times the plasmon energy $h \omega_{p} \sim 10 \mathrm{aV}$.

For phase velocities near the speed of light, the plasina wave numb, ${ }^{\prime}=$ $\omega_{p} / \epsilon \simeq 5 \times 10^{-3} A^{-1}$ is much less than the Fermi waye number $k_{F} \sim 1 A^{-1}$ il the metal, so plasmon damping is primarily due to interband transitions (electron eransitions to unfilled bands) with the decay width $\Gamma_{p}$ being typically 10-2 $10^{-1} \mathrm{hw}_{\mathrm{p}} \sim$ 0.1-1 eV.5 To wse such plasma oscillations in solids to accelerate charged particles to very high energy is problematic since the radiation length for electrons and positrons is of order $1 \mathrm{~cm}$, while the nuelear collision lenglt for protons and antiprotons is of order $10 \mathrm{~cm}$.

These problems can be substantially mitigated for positively charged particles by utilizing the channeling phenomenon in cryatals. ${ }^{6}$ Positively charged particles are guided by the average electric fields produced by the atomic rows or planes in the crystal. The particles make a series of glancing collisions with many 
atoms and execute classical oscillatory motion along the interatomic channels. ${ }^{T}$ In contrast, negatively cherged particles are attracted by the atomic nuclei and suffer large angle Coulomb scatterings resulting in repid dechanneling. This suggests that it is possible to accelerale positively charged particles on plasma waves for considerable distunces through channels in metallic crystals.

An immediate concern in such an acceleration scheme is beam loss through gradual dechanneling. ${ }^{5}$ The transverse momentum of channeled particles increases due to collisions with electrons in the channel. ${ }^{9}$ Dechanneling occurs when a particle's transverse kinetic energy $K_{\perp}=E \psi^{2} / 2$, where $E=\gamma m c^{2}$ is the toisil particle energy and $\psi$ is the channeling angle, allows it to overcome the channel potential energy barrier $V_{c}\left(\simeq z e 10^{2}-10^{3}\right.$ volts for a particl. of charge ze). This defines the critical channeling angle $\psi_{c}=\left(2 V_{c} / E\right)^{t / 2}$. The increase in the angular divergence per unit length for a channeled particle due to multiple scattering can be written as

$$
\frac{d\left\langle\psi^{2}\right\rangle}{d \ell}=\frac{\psi_{\mathrm{e}}^{2}}{2 \ell_{d}},
$$

where $\ell_{d}=\Lambda E / z e$ is the characteristle dectianneling length. The dechanneling constant $A$ is typically $1-10 \mu \mathrm{m} / \mathrm{MV}$, so high energy particles can channel considerable distances ln a crystal. For example, a $1 \mathrm{TeV}$ proton could channel of order $1 \mathrm{~m}$ in a metallic crystal like tungsten. ${ }^{10}$

Particle dechanneling in a solid state accelerator is modified by the fact that a channel's normalized rms acceptanen $\varepsilon_{\mathrm{cn}}=(1 / 2) \gamma a \psi_{\mathrm{c}}$ increases with energy. Here $a$ is the axial channal radius. This effect can compensate for the increase in the channeled particle emittance due to multiple scattering if the acceleration gradient $G$ is high enough. Our earlier calculation" ${ }^{11}$ did not correctly include this effect resulting in a more pessimistic estimate for particle dechanneling than will be given here. The channel acceptance is the phase space area available to particles for channeling motion. Each channel acts like a smooth focusing accelerator with betatron focusing function (wavelength $/ 2 \pi$ of transverse ascillationg)

$$
\rho_{F}=\left(a^{2} E / 2 V_{c}\right)^{1 / 2}=a / \psi_{c} \quad .
$$

Multiple scattering in a transverse focusing system randomly excites betatron oscillations leading to a growth in the invar:unt (or normalized) rms emittance $\epsilon_{n}=\gamma \varepsilon=\gamma \sigma^{2} / \beta_{F}$, where $\varepsilon$ is the unnormalized rms emiltance, and $\sigma^{2}$ is the spatial divergence. ${ }^{12}$ The ernittance growth per unit length can be written as

$$
\left(\frac{d \varepsilon_{n}}{d t}\right)_{\text {acat2. }}=\frac{7 \hat{\beta}_{F}}{2} \frac{d\left(\psi^{2}\right)}{d \ell}=\frac{z e a \psi_{c}}{4 \Lambda m c^{2}} .
$$


Initially trapped particles remain channeled indefinitely provided that

$$
\left(\frac{d \varepsilon_{\mathrm{n}}}{d \ell}\right)_{\mathrm{scatq} .} \leq \frac{d \varepsilon_{\mathrm{cn}}}{d \ell}=\frac{z e G a \psi_{\mathrm{c}}}{4 m c^{2}}
$$

or equivalently $G \geq \Lambda^{-1}$.

Integration of Eq. (3) yieids for the final emiltance after acceleration from $\gamma_{i}$ to $\gamma$,

$$
\varepsilon_{n j}=\varepsilon_{n i}+\frac{\alpha \psi_{\varepsilon}}{2 \Lambda G} \sqrt{\gamma j}\left(\sqrt{\gamma_{j}}-\sqrt{\gamma_{i}}\right) .
$$

$A+$ high energy $\left(\gamma_{f} \gg \gamma_{i}\right), \epsilon_{n f} \simeq a \psi_{\mathrm{c}} \gamma_{f} / 2 \Lambda G$, and arcelerated particles oscillate asymptotically with mean square amplitudo $\sigma_{f}^{2}=\rho_{F} \varepsilon_{n f} / \gamma f=a^{2} / 2 \Lambda C$ about the channel axis. A charged particle channeling through a crystal naturally emits radiation as it oscillates transwersely in a channel. ${ }^{\text {i3 }}$ The maximum altainable energy in such a channeling accelerator occurs when the radiative energy loss approaches the energy gain from the acceleration gradient. The radiative loss per unit length in a smooth focusing system is given by ${ }^{12}$

$$
\left(\frac{d E}{d \ell}\right)_{\mathrm{rad}}=-\frac{2(z e)^{2}}{3} \sigma^{2}\left(\frac{\gamma}{\beta_{F}}\right)^{4} \text {. }
$$

where $\sigma$ is the rms oscillation amplitude. Using $\sigma-\sigma_{I}$ ealculated above for the amplitude and $V_{c} / \Omega \simeq z e 10^{2} \mathrm{~V} / \lambda$ yieids for the maximum energy

$$
E_{\max }=\left(\frac{m}{m_{p}}\right)^{2}(\Lambda G)^{1 / 2}\left(\frac{G}{z^{3} 100 \mathrm{GV} / \mathrm{cm}}\right)^{1 / 2} 10^{5 \mathrm{TpV}}
$$

where $m_{p}$ is the proton rest mass. ${ }^{14}$

Only for acceleration gradients $G \geq \mathrm{A}^{-1} \div 1-10 \mathrm{GV} / \mathrm{cm}$ will significant bearn fractions remain channeled over long distances in a rrystal. In the case of a longitudinal plasma oscillation, this imples that a large amplitude wave with a gradient of order $100 \mathrm{GV} / \mathrm{cm}$ is desira'sle. This gradient corresponds to an energy density of order $10^{8} \mathrm{~J} / \mathrm{cm}^{3}$. The $\mathrm{f}$ lasma whue would oceupy at most a transverse cross section of several sujuare plasma wavelengths $\left(\sim 10^{-9} \mathrm{~cm}^{2}\right)$ over a long acceleration length in the crystal in order to keep the total energy in the plasma wave small and still maintain a uniform wavefront. Whether the energy contained in the plasma wave is sufficient to thermally damage the crystal depends on the relaxation time for converting plasmon enargy to phonons. 


\section{DISCLAIMER}

This report was prepared as an account of work sponsored by an agency of the United States Government. Neither the United States Governmenl nor any agency thereol, nor any of their employees, makes any warranty, express or implied, or assumes any legal liability or responsibility for the accuracy, completeness, or usefulness of any information, apparatus, product, or process disclosed, or represents that its use would not infringe privatcly owned rights. Reference herein to any specilic commercial product, process, or service by trade name, trademark, manufacturer, or otherwise does not necessarily constitute or imply its endorsement, recommendation, or favoring by the United States Government or any agency thereof. The views and opinions of authors expressed herein do not necessarily state or reflect those of the United Siates Goucrnment or any agency thereof. 
After the original plasma wave $\left(\omega=\omega_{p}, k_{p}=k_{p} \hat{z}\right)$ decays via interband transitions, the excited electron states will in turn decay producing a plasmon gas with wave vectors $\left(|k| \simeq k_{p}\right)$ varying in direction. The plasmon gas can cause additional interband transitions but eventually electron-electrost collisions witt break up the plasmons as electrons are scattered out of synchronism. ${ }^{5}$ The electron collision rate can be written approximately as $\Gamma_{\epsilon e} / \hbar \simeq 0.4\left[\left(k-k_{F}\right) /\left.k_{F}\right|^{2} E_{F} / \hbar\right.$ when the electron wave number $k$ is near the Fermi wavenumber $k_{F}$. ${ }^{15}$ Here $E_{F}$ is the Fermi energy which is typically less than the plasmon energy $h w_{p}$. Electrons in a plasmon have wave numberg $k \sim k_{F}+k_{p} / 2$, so the plasmon gas decays into a hot electron gas in about $10^{-10} \mathrm{sec}$. These superthermal electrons have energies of order $10^{8} \mathrm{~J} \mathrm{~cm}-3 / 10^{22} \mathrm{~cm}^{-3} \sim 100 \mathrm{keV}^{-}$but lose their energy at a rate of about $1 \mathrm{MeV} / \mathrm{cm}$ primarily through plasmon radiation and electron collisions, This distributes the energy of the original plasma wave radially about $1 \mathrm{~mm}$ among many thermal electrons which then heat the crystal by phonon emission $\left(r_{e}-\sim\right.$ phonon $\left.\sim 10^{-14}-10^{-15} \mathrm{gec}\right)$. The plasma wave energy density thus decreases to about $10 \mathrm{~J} / \mathrm{cm}^{3}$ in $10^{-10} \mathrm{sec}$ corresponding to a tolerable power input of $10^{13}$ $W / \mathrm{cm}^{3}$ to the lattice. Crystal damage would occur for power inputs of ordes $10^{13} \mathrm{~W} / \mathrm{cm}^{3}$ in a $10^{-10} \mathrm{sec}$ pulse..$^{16}$

The generation of large amplitude plasma waves in a metal presumably requires an intense power source to oupply the plasma wave energy in a short time without destroying the crystal. Certainly creative ideas for exciting such waves in a metal are needed. We brieliy consider three possibilities, all of which are at best problematic when applied to metallic electron plasmas.

The laser beat-wave method ${ }^{37}$ involves resonantly exciting the plasma wave by the ponderomolive force of two collinear beating lasers with frequency difference $\omega_{1}-\omega_{2} \simeq \omega_{p}$. In a metal this requires $X$-ray lasers with $\omega_{1, j} \geq 10^{17} \mathrm{sec}^{-1}$. The plasmon decay width $\Gamma_{p}$ results in the wave saturating at an amplitude $\alpha_{p} \equiv e \varepsilon_{p} / m \omega_{p} c \simeq \alpha_{1} \alpha_{2} h \omega_{p} / 2 \Gamma_{p}$, where $\alpha_{i}=e \varepsilon_{i} / m \omega_{i} c$ are the normalized laner fields. To obtain a plasma wave with $\alpha_{p} \sim 10^{-2}-1$ requires $\alpha_{1} \alpha_{2} \geq 10^{-4}-10^{-2}$ or a laser intensity $I \gtrsim 10^{17}-10^{19} \mathrm{~W} / \mathrm{cm}^{2}$. Since this intensity is to be delivered in a $10^{-14} \mathrm{sec}$ pulse with a $10^{-9} \mathrm{~cm}^{2}$ spot size, crystal survivability it questionable.

An immediate problem with beat-wave excitation is pump depletion as the lasers leave their energy behind in plasma waves. The laser-acoustic wave scheme avoids this problem by side-injecting a laser with frequency $\omega_{0} \simeq \omega_{p}$ into a plasma containing an acoustic wave. ${ }^{3 \theta}$ The laser is linearly pola, ised along the direction of the acoustic wave vector. The laser $\left(\omega_{0}, k_{0}\right)$ and acoustic wave ( $\omega_{a c}, \bar{k}_{a c}$ ) quasiresonantly excite lorward and backward traveling plasma waves with $\omega=\omega_{\mathrm{a}}+w_{\mathrm{ac}} \simeq \omega_{\mathrm{b}}$ and $k_{\mathrm{p}}=k_{\mathrm{c}} \pm k_{\mathrm{dc}} \simeq \pm \bar{k}_{\mathrm{ac}}$. In a metal the plasma wave 
saturates at an amplitude $\omega_{p} \simeq \alpha_{0}\left(\delta n_{0 F} / n_{0}\right) \hbar \omega_{p} / 2 \Gamma_{p}$ whore ro is the normalized laser ficld and $\delta n_{a c} / n_{n}$ is the acoustic wave density perturbation. To excite a plasma wave with $\alpha_{p} \sim 10^{-2} \quad 1$ requires $\alpha_{0} \delta n_{a c} / n_{n} \geq 10^{-1}-10^{-2}$ corresponding to an ultraviolet lasen intentsity of $10^{15}-10^{17} \mathrm{~W} / \mathrm{cm}^{2}$ if $\mathrm{mon}-10^{-2}-10^{-1}$. The crystal may survive this high intensity because the pmergy would be primarily absorbed in plasmons and interband transitions nnt only later converted to lattice heat as discussed earlier.

The wakefield urethod for exciting plasma waves eliminates the need for lasers by employing a chargnd relativistic driving beam to leave behind a wake of plasma waves. 19 The ratio of the maximum nccelerating wakefield to the maximum decelerating field experienced by the driver is called the transformer ratio, $R$ : $\left|\varepsilon^{+} / \varepsilon^{-}\right|$. For a nonsymmetric finite leng:h driver, $R$ can be arbitrarily large. ${ }^{20}$ In a metal collisional energy loss (1-10 MeV/cm) of the driver to electrons may destroy the crystal as the thermal elcctrons rapidly $\left(10^{-14}-10^{-15} \mathrm{sec}\right)$ heat the lattice by phonon emission. To excite a plasma wave with $\alpha_{p} \sim 10^{-2}-1$ recuires a driver charge density of order $10^{20}-10^{22} \mathrm{e} / \mathrm{cm}^{3}$. This yiclds a power inpur to the lattice of order $10^{18}-10^{20} \mathrm{~W} / \mathrm{cm}^{3}$.

Independent of the method for exciting a plasma wave, similar considerations apply to the collisional energy loss by the accelerated beam. The thermal Tracture threshold will presumably Jimit the maximum accelerated bean current dengity that the crystal can withstand to appioximately $10^{\prime \prime} \mathrm{A} / \mathrm{cm}^{2}$ for a pulse length of order $h / \Gamma_{p} \sim 10^{-14} \mathrm{sec}$. Although the channeling phenomenon and high acceleration gradient aid in maintaining the accelerated beam emittance over long distances, the collisional energy loss is a consequence of the collective nature of this solid state acceleration scheme. Certainly the scheme explored in this paper does not preclude other possibilities for accelerating particles in solids.

While completing the work described here, we discovered that several authors have discussed the acceleration of channeled particles by various types of fields in solids. We briefly mention these papers for the interested reader but do not clain the list to be complete. Kanofsky ${ }^{21}$ discussed the use of masked laser fields to accelerate particles in a crystal and presented two bean dechanneling estimates. One of these estimates was identical to our more pessimistic dechanneling calculation in Ref. 11 which did not included the adiabatic damping of the beam emitlance. Grishacv and Nasonov ${ }^{22}$ suggested the acceleration of particles on a longitudinally polarized wave in a cubic crystal having a nonlinear optical susceptibility. Pisarev ${ }^{23}$ analyzed the use of the longitudiral static polarization produced in a nonlinear crystal by optical waves to accelerate particles. Neither of these papers contained a discussion of dechanneling. Beloshitskii and Kumakhov ${ }^{24}$ considered the use of the inverse Cerenkov ffect in a rrystal to 
accelerate particles with a laser. Besm dechanneling was briefly discussed in this paper with the ouggeation that the decrease in beam enittance with increasing energy could trop channeled particies. Nesonov's5 suggested that particles could be accelerated by longitudinal optical phonons in an alkalf halide crystal but did not mention dechanneling. In all these papera, the maximum acceleration grodients were estimated to be 0.1-1 $\mathrm{cV} / \mathrm{cm}$.

\section{ACKNOWLPDGEMENTS}

The authors would like to thank R. Bruinsma, G. Grûner, L. Mihaly and H. Kruger for informntive diacusslons about erystals and solids.

\section{REFBRENCES}

1. Laster Aeceleralion of Partieles (Los Alamos, 1982), P. J. Channel, ed., A.I.P. Conf. Proc, No. 91 (A.I.P., New York, 1982).

2. Lasef Aceeleration of Partieles (Malibu, CA, 1985), C. Joshi and T. Katgoulans, eda., A.I.P. Conf. Proc. No. 130 (A.l.P., New York, 1985).

3. T. Tajime and J. M. Dewson, Phys, Rev. Lett. 43, 267 (1979).

4. C. J. Powell and J. B. Swan, Phys. Rov. 115, 869 (1959). N. H. March and M. Parrinello, Colluetive Efects in Solids and Liquids (Adam Higler Ltd., Bristol, UK, 1982).

B. P. C. Gibbona et al., Phys. Rav. B13, 2451 (1976), P. C. Gibbons, Phys. Rev, B25, 2356 (1981).

6. D. S, Gemmel, Rov. Mod. Phys. 4B, 129 (1974). Y.-H. Ohtsuki, Charged Beam Interaetions with Solids (Taylor and Franeig, New York, 1983).

7. The condition for clasalcal motion is that the trangverse de Broglie wavelength $h / p \psi$. where $\psi$ in the incidont angle to s row or plane, be much less thitn the typienl atomle screening length $(\sim 0.1 \mathrm{~A})$ where single atomic collisions become important. At this critical distance, the channeling potential prergy $V=V_{t}$.

8. F. Bonderup et al., Rad. Effects 12, 261 (1972).

9. The collisional energy lose for relativistic particles in solids is typically $(d E / d l)_{e}=1-10 z^{2} \mathrm{MV} / \mathrm{cm}$. We assume the acceleration gradient $G$ satisfies se $G>(d E / d Q)$.

10. R. A. Carrigan el al, Nucl. Inatrum. Methodo 104, 205 (1982).

11. P. Chen and R. J. Noble, in A.I.P. Conf. Proc. "Symposium on Advanced Accelerator Concepts" (Madison, Wis., Aug. 1986, eds. D. Cline and F. Mi11s). 
12. B. W. Montague and W. Schnell, Ref, 2, p, 146.

13. M. A. Kumakhov, Phys. Lett. D57, 17 (1976); Phys, Stat. Sol. B84, 41 (1977). V. V. Beloshitsky and F. F. Komarov, Physies Reporto 93, 127 (1982).

14. This anstyais neglects radiative codiug of the emittance which can act againgt the effect of multiple scattering.

15. J. J. Quinn, Phys. Rev. 129, 1453 (1962). L. Hodin and 5. Lundqvist, in Solid State Phygies, F. Seita, D. Turnball and H. Bhronreich, ods. (Acadamic, New York, 1969), Vol. 23.

16. This is the fracture threshold due to thermal yhock. For timen lass than the characteristic time for acoustic waves $\left(v_{d t} \approx 10^{6} \mathrm{~cm} / \mathrm{sec}\right)$ to remove energy from a given volume, metals have a dynamie tonsile otronglh of soveral kilobars. The change in pressure $P$ per unit onergy $U$ al comatant volume $V$ is $V(d P / d U)_{V} \sim 10^{-2}$ kilohar $/ J / c \pi 1^{1}$ in melak.

17. T. Tajima and J. M. Dawson, IEEE Trans. Nucl. Sei. NE 28, 3416 (1081).

18. T. Katsouleas at al., IEEF Trans. Nucl. Sci. NS 32, 7554 (1783).

19. P. Chen et al., Phys. Rev, Lett. 54, 693 (1045).

20. K. I. F. Bane, P. Chen and P. B. Wilson, IEEE, Trans. Nurl. Sai, NS 32 , $3524(1985)$.

21. A. Kanofsky, Rev. Sci. lnst. 48, 34 (1977).

22. I. A. Grishaev and N. N. Nasonov, Pis'ms Zh. Tekh. Fiz, A, 10A4 (1877) [Sov. Tech. Phys. L,ett. 3, 446 (1977)].

23. A. F. Pisarev, Zh. Tekh. Fiz. 40, 786 (1079) |Sov. Phys. Treh. Phys, 24, 456 (1979)\}.

24. V. V. Beloshitskii and M. A. Kumakhov, Jokl. Akod. Nouk SSSR 249, 100 (1979) [Sov. Phys. Dokl. 24, 916 (1979)].

25. N. N. Nasonov, Pis"ma 2h. Tekh. Fiz, B, 100 (10R0) Jov, T'reh. Phyg. Lall. $6,214(1980)]$. 